How to reference this article

Boni, F. (2021). Tracce veneziane in un manoscritto appartenuto a Maria Casimira Sobieska. Tra Cabala e magia naturale. Italica Wratislaviensia, 12(1), 87-105.

DOI: http://dx.doi.org/10.15804/IW.2021.12.1.05

Fabio Boni

Uniwersytet Pedagogiczny im. KEN w Krakowie, Polonia

fabio.boni@up.krakow.pl

ORCID: 0000-0001-5977-7138

\title{
TRACCE VENEZIANE IN UN MANOSCRITTO APPARTENUTO A MARIA CASIMIRA SOBIESKA. TRA CABALA E MAGIA NATURALE
}

\author{
BETWEEN KABBALAH AND NATURAL MAGIC: \\ VENETIAN TRACES IN A MANUSCRIPT THAT \\ BELONGED TO MARIA CASIMIRA SOBIESKA
}

\begin{abstract}
The article presents some texts from manuscript 2284 stored at the Jagiellonian University Library in Krakow, titled Księga Kabat Królowej Sobieskiej (The Kabbalah Book of Queen Sobieska). It is a collection of unpublished texts about magic, Kabbalah, and astrology, collected by Maria Casimira Sobieska herself during her long sojourn in Italy (1699-1714). Some of the manuscript's texts were written in Italian, most likely in Venice at the end of the $17^{\text {th }}$ century, and signed by a certain Andrea Valetta, a citizen of the Most Serene Republic. Some of these texts concern natural magic, and others, Kabbalah: Acqua simpatica per scivere [sic] da lontano, Per fare l'acqua ardente, A' far la scrittura simpatica, and Altra Acqua simpatica (104r-105v); Modo di scrivere in Zifra senza dar sospetto di Zifra and Tabella della prima scienza numerica (100r); Seconda scienza numerica (100v-101v); Trattato Per estrarre il nome del Genio (106r-113r); Regole di Cabala di Salomone Imparatale divinam [ente] da Dio (216r-222v). The work shows how, in the texts on natural magic, Valetta follows Giovanni Battista Della Porta's theory and approach to natural magic. In the texts on Kabbalah, although he considers Kabbalah from a Christian point of view, like Pico della Mirandola, he ignores mystical and spiritual aspects — which, in Pico, are most important-focusing on practical and prophetic aspects.
\end{abstract}

Keywords: Maria Casimira Sobieska, Venice, Kabbalah, natural magic, $17^{\text {th }}-18^{\text {th }}$ century 
【1 manoscritto 2284, conservato nella Biblioteca Jagellonica di Cra1 covia, conosciuto con il titolo di Księga Kabat Królowej Sobieskiej (Libro della Cabala della Regina Sobieska), contiene diversi testi a carattere cabalistico, magico ed astrologico ${ }^{1}$, probabilmente raccolti, o fatti trascrivere, dalla regina Maria Casimira d'Arquien Sobieska (1641-1716) durante il suo lungo soggiorno italiano (1699-1714)2. Diversi testi sono inoltre di mano della regina stessa, redatti in lingua italiana e francese ${ }^{3}$.

Alla Biblioteca Jagellonica sarebbe giunto intorno al 1823, grazie alla donazione del conte Ignacy Morski, il quale lo avrebbe acquistato durante un viaggio a Roma con la moglie negli anni 1792-1793. Esso consiste in un blocco di testi, il cui numero di carte ammonta a 246, l'impaginazione è databile all'inizio del secolo XIX (Wisłocki, 1881, p. 546; Pietrzak, 2016, p. 70; Sosnowski, 2017).

Alla carta $85 \mathrm{v}$ del manoscritto troviamo quella che potrebbe essere una nota di possesso, da mettere in relazione con diversi testi del manoscritto stesso, la quale recita:

Andrea Valetta S. Samuel

in Calle Cà Mocenigo

1 Il numero totale dei testi è di 55, redatti in diverse lingue e da diverse mani, da datare individualmente: italiano (22), francese (22), latino (10), tedesco (1). Per un'analisi approfondita dei testi in lingua italiana e del manoscritto stesso vedi ŚnieżyńskaStolot \& Boni.

2 La vedova di Jan Sobieski, dopo avere lasciato la Polonia il 2 ottobre 1698, entrò infatti in Italia il 5 gennaio dell'anno successivo, accolta a Verona da Ferdinando Gonzaga, Rinaldo d'Este e Francesco Farnese. Successivamente, il 18 dello stesso mese, fece la sua entrata a Venezia, dove incontrò Marco d'Aviano, trattenendosi nella città lagunare per venti giorni, prima di riprendere il suo viaggio verso Roma, meta finale e poi suo luogo di residenza per i successivi quindici anni (Platania, 2018, pp. 10-11).

3 Tra i testi in italiano si possono menzionare ricette per la preparazione di cosmetici o medicinali, come alle c. 133v-134r "Bianco per la faccia", 135v "Aqua [sic] per imbiancare il viso", 142v "Modo di far perdere forza al tabacco", 215v "Saponete ligiere di schiuma", ma anche appunti per procedimenti cabalistici, come "Cabala Curiosa" (c. 226-228v) o "Modo scientifico da sapersi l'esito d'una persona per mezzo del [sic] prima lettera del suo proprio nome et ultima dell'istesso" (c. 169r-170r). Anche i testi in francese trattano tematiche simili. Per una completa descrizione del manoscritto e delle sue varie parti cf. Sosnowski, 2017 e Śnieżyńska-Stolot \& Boni. 
Da questa breve annotazione si può affermare l'origine veneziana di colui che l'ha vergata e ipotizzare un'attività di copista di professione o di appassionato di scienze occulte dello stesso. Il riferimento a San Samuel potrebbe far pensare a un suo legame con l'omonimo teatro, in attività dal 1656, o col sestiere di San Marco, dove si trova tra l'altro la chiesa di San Samuele, poco distante da Calle Mocenigo, indicata come indirizzo dell'autore.

A partire da una data che appare in un testo da lui composto possiamo inoltre stabilire che sia vissuto nella seconda metà o verso la fine del secolo XVII. La data in questione è il 1685, come figura alla c. $100 \mathrm{v}$ in relazione a un quesito a cui cercare risposta attraverso il metodo cabalistico: "An Barbara sit Fidelis Tomae Portio l'Anno 1685". A conferma del fatto che l'autore fosse ancora in vita dopo tale data si può inoltre portare un altro riferimento interno ai testi riconducibili alla sua mano, presente alla carta $113 \mathrm{v}$ : "si ricerchi qual sarà la fortuna di Guglielmo d'Oranges hora Rè d'In[ghilterra]". Guglielmo d'Orange (1650-1702) fu infatti re d'Inghilterra e Scozia dal 1689 all'8 marzo 1702, arco temporale in cui quindi tale nota veniva scritta. Non sarebbe forse neppure da escludere che l'autore fosse ancora in vita nel 1699, anno in cui Maria Casimira entrò a Venezia: si potrebbe anche ipotizzare che la regina avesse ordinato all'autore la trascrizione dei testi (o che li avesse da questi acquistati), che poi avrebbe portato con sé a Roma e avrebbero costituito parte dei suoi appunti cabalistici.

L'annotazione alla carta $85 \mathrm{v}$ è assai importante, poiché permette di identificare in maniera sicura, sulla base di un confronto grafico, i testi redatti da Valetta. Caratteristiche della sua scrittura sono in particolare la grafia della lettera "c" in unione con vocale all'interno di una parola e la stessa a inizio di parola, con la presenza di un evidente fronzolo nella parte superiore e inferiore della lettera; la "s" maiuscola ad inizio di parola; il nesso "nd" all'interno di parola; la forma della stessa "d" con tipico fronzolo verticale curvato e rientrante verso sinistra.

I testi in cui si riconosce la mano di Andrea Valetta si legano a due temi ben definiti: la magia naturale e la cabala. Gli scritti riguardanti la magia naturale comprendono ricette e medicamenti, nonché tecniche 
di scrittura simpatica e nascosta (steganografia) ${ }^{4}$ : Si facci uno spirito di vitriolo d'Ungaria (93r-93v); Acqua simpatica per scivere [sic] da lontano, Per fare l'acqua ardente, A' far la scrittura simpatica, Altra Acqua simpatica (104r-105v); A fare il Vero oglio di Talco senza additione di cosa alcuna (140r-141r); Modo di scrivere in Zifra senza dar sospetto di Zifra (224r). I testi cabalistici comprendono invece istruzioni e spiegazioni per ottenere risposte a un dato quesito sulla base dei valori numerici attribuiti a ciascuna lettera dell'alfabeto che compone le parole del quesito, attraverso la costruzione di piramidi e di quadrati di numeri, nonché un testo dedicato ai procedimenti da seguire per scoprire il nome del proprio genio tutelare: Tabella della prima scienza numerica (100r); Seconda scienza numerica $(100 \mathrm{v}-101 \mathrm{v})$; Trattato Per estrarre il nome del Genio (106r-113r); Per mettere in opera la consaputa Cabala numerica (113v-121v); [Tabella cabalistica] Regola (122r-123r); Capo Primo. Questa scienza viene chiamata Cabala (171r-193r); Regole di Cabala di Salomone Imparatale divinam [ente] da Dio (216r-222v).

Non essendo qui possibile prendere in esame e approfondire tutti questi testi, vorremmo soffermarci soltanto su alcuni di essi, rappresentanti dei due ambiti menzionati, per provare a porne in evidenza le peculiarità, al fine di individuare il contesto culturale con cui poterli mettere in relazione e risalire così alle fonti, o autorità, che potrebbero averli ispirati e al rapporto dell'autore con esse.

In alcune delle ricette proposte da Valetta si possono scorgere influssi legati alla concezione della magia naturale, la quale aveva avuto il suo più florido sviluppo nel Rinascimento. Pensatori come Pico della Mirandola, Marsilio Ficino, Cornelio Agrippa, vedevano in essa la possibilità di intervenire sulla realtà naturale, penetrandone i più reconditi e occulti segreti. Il "mago" in questa accezione era colui che sapeva osservare la natura e cogliere la trama di legami che univa tutte le cose, conoscendone le proprietà segrete, così da poterne ricreare i fenomeni e influire su di essi, impiegandoli a vantaggio dell'essere umano. Non si trattava

4 La steganografia, letteralmente "scrittura coperta", era una tecnica utilizzata per criptare le informazioni contenute in un testo e assicurarne così la segretezza (Eco, 2002, p. 207). 
più quindi di un atteggiamento meramente contemplativo dell'uomo di fronte alla meraviglia della natura, come era stato nel Medioevo, ma di una volontà di operare su di essa servendosi di una conoscenza pratica, sebbene alla portata di pochi iniziati (Yates, 2019, p. 160).

La magia naturale da un punto di vista pratico ${ }^{5}$ si basava sostanzialmente sul concetto che in ogni cosa si trovasse una parte di ogni altra cosa. Da ciò discendevano due principi generali, ovvero che il simile produce il simile (principio della somiglianza) e che le cose che in qualche modo sono state generate insieme mantengono un legame tra loro (principio del contatto) e si influenzano a vicenda anche una volta separate e lontane (Dobrowolski, 1990, p. 57). Le proprietà occulte da cui tutte le cose sono attraversate riguardano quindi accordo (simpatia) e disaccordo (antipatia): tutte le cose si attraggono e si respingono, compito del mago è quello di ricercare e distinguere queste proprietà (ibid., p. 68).

Nell'ambito dell'applicazione pratica della magia naturale, fu Giovanni Battista Della Porta (1535-1615) a distinguersi per il suo impegno sperimentale, prima ancora che teorico, con la pubblicazione in latino di quello che si può definire un manuale di magia naturale, intitolato Magiae naturalis sive de miraculis rerum naturalium, uscito una prima volta nel 1561 ad Anversa e in traduzione italiana, a Venezia, nel 1567 col titolo De i Miracoli et maravigliosi effetti dalla natura prodotti Libri IV; successivamente un'edizione ampliata, in latino, vide la luce a Napoli nel 1584 e, più tardi, in italiano, a cura di Pompeo Sarnelli, nel 1677, col titolo di Della Magia naturale del Signor Giovan Battista Della Porta napolitano. Libri XX. Della Porta si occupò tra l'altro anche di arte della memoria, di scrittura cifrata con l'opera De furtivis literarum notis vulgo de Ziferis (1563), nonché di fisiognomica (De humana physiognomia, 1586). Egli considera la magia naturale come sapienza e conoscenza perfetta della natura e fa rientrare nel suo campo anche medicina, biologia, ottica (ovvero la fisica), nonché l'alchimia. Il mago,

\footnotetext{
5 Tralasciamo qui le questioni più propriamente filosofiche che si legano all'ermetismo e al neoplatonismo rinascimentale, per cui si rimanda a Yates, 2019, in particolare i cap. I-VIII.
} 
per definirsi tale, deve conoscere queste scienze, di cui la magia naturale è il coronamento: "apex naturalium scientiarum" (Della Porta, 1584, p. 45; Dobrowolski, 1990, p. 63). L'accento naturale riferito alla magia voleva significare che tale tipo di pratica si inseriva all'interno dei meccanismi della natura e del cosmo, ovvero escludeva l'intervento e la mediazione di forze soprannaturali. Proprio le qualità spirituali delle cose, che permangono occulte, rappresentano il campo di ricerca del mago naturale, poiché la loro conoscenza costituisce la chiave per intervenire sul mondo.

Della Porta era convinto dell'importanza, per la ricerca delle qualitates occultae, della pesatura e in particolare della distillazione, a cui aveva dedicato il libro IX della Magia naturalis, De extrahendis rerum essentiis, e l'opera De distillationibus libri IX (1609). Attraverso la distillazione, alla cui base sta la capacità di regolare l'intensità dell'elemento del fuoco, si può ottenere una sostanza materiale che costituisce l'essenza nascosta di ciascuna cosa sottoposta a tale processo. L'obiettivo di ogni procedimento di distillazione era quello di estrarre la quinta essenza, ovvero l'essenza nascosta di tutte le cose materiali (Dobrowolski, 1990, p. 89). Grazie alla distillazione e all'ottenimento della quinta essenza era così possibile preparare medicinali o altre sostanze purissime da piante, radici, ma anche minerali e metalli. Tali processi di trasformazione della materia dovevano portare, nel loro risultato più alto, alla creazione dell'elisir di vita, altrimenti detto panaceum, in grado di guarire tutti i mali, e al permutamento dei metalli meno nobili in argento e oro. L'alchimia, la scienza della distillazione, era per Della Porta una scienza occulta, nel senso che era accessibile soltanto a chi fosse stato in grado di penetrare i segreti del grande laboratorio della natura e di riprodurli nel proprio: questi era lo scienziato-mago naturale (ibid., pp. 94-95).

Dopo questa breve panoramica sulla magia naturale, possiamo avvicinarci ad alcune ricette di Valetta, per osservarne le particolarità e vedere se possono essere in qualche modo messe in relazione con le concezioni di Della Porta sulla magia naturale.

A questo proposito pare interessante considerare il procedimento che il veneziano fornisce per ottenere l'Acqua simpatica per scivere 
[sic] da lontano, che si trova alle carte 104r-105r del manoscritto. Leggiamo la parte iniziale del procedimento:

Rq [requiritur] calce viva q[uantum]. V[is] esponi all'Aria in tempo sereno sino che si faccia in polvere, mettila in storta luttata, e ben chiuse le giunture del Recipiente, si distilli à fuoco forte sino che più non distilla, e di quest acqua se nè facci qualche quantità, cioè una libra se si può.

Poi si facci spirito volatile di vitriolo ungarico, che gettandone in Aria non cada in terra.

Poi Rq. [requiritur] di detta acqua e del spirito volatile di vitriolo una parte eguale se nè faccia unione e si distilli per una sola volta, et il distillato si ponga à parte.

Poi habbi qualche quantità di sangue humano di huomini di pelo rosso se si può, mà robusti ò in una volta, ò in più volte, e cavato, che sarà sì lascia condensare, poi si mette in un sachetto di tela bianca, e si dimeni in acqua chiara sino che l'acqua più non si tinge, doppo si apra il sacchetto, e si raccoglieranno diligentem[ente] quei fili bianchi che vi saranno, che sono le sole fibre del sangue, quali poi si mettono sopra un foglio di carta ad asciuttarsi all'Aria, e di questi fili si procurerà farne qualche buona dose.

Poi si piglia una parte di detti fili, e del detto spirito ultimo distillato parti trè, si metta ogni cosa dentro un sagiolo, e sia benissimo sigillato, e si metta in fimo di cavallo per giorni quaranta, doppo cava il detto spirito, e mettilo in un orinale e col suo cappello e Recipiente e ben chiuse le giunture, si distilli à B.[agno] M.[aria] à siccità e si troverà nel vaso un sale bellissimo cristallino. $(104 \mathrm{r}-104 \mathrm{v})$.

Possiamo già osservare alcuni aspetti che rimandano agli insegnamenti dellaportiani sulla distillazione, come il processo di trasformazione e trasmutazione della materia per ottenerne l'essenza. In particolare qui, attraverso una sapiente regolazione del fuoco, a partire dalla distillazione della calce viva e del vetriolo, passando per il disseccamento di sangue umano, e facendo ancora circolare il tutto in distillazione, si giunge alla formazione di un "sale bellissimo cristallino". Vale la pena notare anche la distillazione in "fimo" e all'aria, grazie all'intervento del sole, ricordate da Della Porta come possibili metodi distillatori nel libro X della Magia naturale, al capitolo IV, Come si distilli al sole (Della Porta, 1677, p. 338). Nel medesimo libro, al capitolo XIV, Come 
si debbano cavare i sali dalle cose, aveva poi lodato le proprietà dei sali ottenuti tramite l'unione e la distillazione dei "semplici" per mezzo del fuoco: "è stata gran tempo questione [...] se i sali ritengono le virtù, che avevano i loro semplici prima, o vero per la forza del fuoco altre ne perdano, altre ne acquistino, $[\ldots]$ si vede che conservano alcune virtù de' semplici, da' quali sono stati cavati, e molte altre assai più acute." (Della Porta, 1677, p. 362) ${ }^{6}$. Vedremo, proseguendo nella preparazione, quali virtù questo sale così ottenuto contenga. Prima di procedere oltre, è però necessario soffermarci sul particolare, a prima vista curioso, riguardante l'ingrediente del sangue umano ${ }^{7}$ di uomo dal pelo rosso e di costituzione robusta. La presenza di questo ingrediente pone la ricetta nuovamente nell'alveo della magia naturale, in quanto si richiede il sangue di un uomo nato sotto l'influsso positivo del pianeta Giove: il colore rosso dei capelli, l'incarnato roseo e la costituzione robusta erano infatti caratteristiche dell'uomo "gioviale" (Śnieżyńska-Stolot, 2017, p. 25), Giove inoltre era un pianeta vivificante secondo la concezione di Marsilio Ficino e il rosso era il colore che ne veicolava gli influssi benefici (Yates, 2019, p. 70). Anche lo stesso Della Porta nella sua Physiognomia indicava proprio negli uomini dalla carnagione vermiglia e di corporatura forte i depositari del sangue più perfetto, in quanto "sanguigni" (Della Porta, 1610, p. 17).

La fase successiva della preparazione consiste in una ulteriore distillazione del prodotto precedentemente ottenuto, unito allo spirito di vino, per ottenere una sostanza liquida, ancora più pura, che servirà poi nell'ultima fase del procedimento, "l'acqua ardente":

Rq. [requiritur] il quarto d'una boccia da lira di spirito di vino perfettiss[imo] che sarebbero libre due, peso medicinale, metti in storta di vetro con la quan-

${ }^{6}$ Citiamo dalla traduzione della Magia naturale, 1677.

7 Il sangue umano, così come altre sostanze di provenienza corporea, era uno degli ingredienti più preziosi e ritenuti più efficaci non solo nei processi alchemici, ma anche nelle ricette mediche: seguendo il concetto "homo homini salus [...] il corpo umano $[\ldots]$ poteva produrre utili frutti terapeutici: i suoi escrementi [...] offrivano al medico buone munizioni, ottimi strumenti d'intervento salutare", all'apice di questi ingredienti stava la "quinta essenza del sangue umano" (Camporesi, 1997, pp. 39-40). 
tità d'un pisello del d[etto] sale, e distilla à foco lento, e passerà ogni cosa nel recipiente $[\ldots]$ sino che è tutto disciolto e passato in spirito, allora sarà l'acqua ardente preparata. $(104 \mathrm{v})$

Possiamo anche in questo passaggio osservare l'importanza, già evidenziata da Della Porta, del peso nel processo di distillazione per giungere alla conoscenza (e all'utilizzo) delle proprietà nascoste delle cose e, ancora una volta, la sapiente regolazione del fuoco per ottenere la trasmutazione della sostanza e ricavarne l'essenza.

Dopo la preparazione di questo ingrediente, si giunge così alla fase finale, che potremmo chiamare dell'esperienza, "A' far la scrittura simpatica":

Si bagna una spognia con[tenente] spirito ardente se ne bagna la carta da scrivere, e bagnata si lasci asciugare da sé.

Poi recipe inchiostro ord[inario] parti dieci e del spirito sud[etto] parte una, e poi questo inchiostro si divida, assieme anco la carta sud[etta] preparata si dividano le persone, che la vogliono sperimentare nel scriversi una all'altra, in che lontananza più piace, assegniandosi il giorno è l'ora, in cui si dovrà scrivere, e scrivendo una persona all'altra nell'hora designata osserverà, quella che aspetta dall'amico saper qualche cosa, osserverà la sua carta senza scrivere, se quel tanto, che il suo amico le può haver scritto da longi sopra l'altra metà di quel foglio, che frà di loro restò diviso, se veramente la vede comparire sopra quella metà di foglio che à quest effetto have sotto gl'occhi la qual cosa dovrà senza dubio succedere con particolar ammira[zione] e stupore.

N.[ota] B.[ene] il sale sud[etto] è per se solo una somma medicina datone un mezo grano in acqua [guarisce] in tutti li mali. (105r)

Possiamo comprendere il funzionamento di questa scrittura simpatica a partire dai principi su cui si basa la magia naturale menzionati in precedenza, ovvero somiglianza e contatto. La carta e l'inchiostro contengono in sé la medesima sostanza, le cui proprietà si conservano anche una volta che vengono separati e distribuiti ai partecipanti. È bene notare che anche questi sono legati tra loro da un vincolo, che è quello della conoscenza e dell'amicizia. Inoltre, ingrediente fondamentale della sostanza finale ricavata è il sangue umano, ulteriore collegamento tra le 
persone, le quali non si riuniscono in un momento qualsiasi, ma in un'ora e in un tempo precedentemente insieme concordati. Siamo quindi di fronte ad un processo di concatenazione, in cui ogni anello della catena è legato a quello successivo. È ciò che Della Porta, nella sua concezione di magia naturale, definiva concatenatio, una delle leggi della natura, secondo cui tutte le cose e i fenomeni sono uniti in un'unica totalità: come in una ruota, messo in moto il primo ingranaggio, tutti i successivi fenomeni si verificheranno da sé, naturalmente, fino all'ottenimento di quello finale, ricercato (Dobrowolski, 1990, p. 121). Un altro risultato di questo procedimento, non meno importante, riguarda proprio quel sale "bellissimo cristallino", i cui granelli sembrano costituire quello che Della Porta poneva all'apice delle sue ricerche, accanto alla quinta essenza, quel panaceum contro tutte le malattie.

A margine di queste considerazioni, possiamo osservare come questo tipo di scrittura ricercata da Valetta abbia uno scopo eminentemente pratico e riveli il suo interesse per la scrittura nascosta.

Passiamo ora al secondo ambito a cui si vorrebbe ancora accennare, legato ai testi di mano di Valetta, ovvero quello cabalistico.

La Cabala ebraica, che ebbe il suo apogeo in Europa nel secolo $\mathrm{XIII}^{8}$, ricerca nella Torah scritta la Torah eterna, precedente la creazione del mondo e trasmessa da Dio agli angeli. Il cabalista tratta quindi il testo sacro come un insieme di simboli che portano con sé un significato mistico e metafisico, e che è necessario decifrare. Per penetrare questo senso più profondo del testo si ricorre a un'opera di scomposizione e ricomposizione del testo stesso, attraverso tre procedimenti: del notarikon (la tecnica dell'acrostico, ovvero l'analisi delle prime lettere di ogni parola), della gematria (ad ogni lettera dell'alfabeto ebraico corrisponde una cifra e quindi ogni parola possiede un determinato valore numerico dato dalla somma dei numeri corrispondenti ad ogni lettera che la compone) e della temura (l'arte del permutamento delle lettere

8 È questo il periodo in cui Moses de León (1250-1305) diffonde in Spagna quello che diviene il testo di riferimento per la tradizione cabalistica, lo Zohar ("Libro dello splendore"). Attribuito al rabbino Simon Bar Jochai, vissuto nel II secolo, sarebbe stato in realtà redatto proprio da Moses de León in aramaico (Ouaknin, 2006, p. 30; Scholem, 2007, p. 180). 
e dell'anagramma). Quest'ultima tecnica è quella di cui Dio stesso si è servito per la creazione del mondo, attraverso la combinazione delle lettere dell'alfabeto, 22, più le 10 Sefirot, ovvero le sue emanazioni. L'alfabeto dà quindi un'infinita possibilità di combinazioni e la lettura della Torah in questo senso porta a un risultato estatico: scomponendone e ricomponendone le singole parole, attraverso le loro lettere, si decifrano e recitano i nomi di Dio. La "cabala dei nomi" fu in particolare sviluppata da Abraham Abulafia (1240-1291), per il quale ogni singola lettera dell'alfabeto ebraico possiede già in sé un significato ed è un nome di Dio. La recitazione di tali lettere, oltre a condurre a un effetto d'estasi, portava anche a ottenere una potenza magica, in quanto quelle lettere articolate in suoni erano le stesse di cui si era servito Dio nella creazione del mondo (Eco, 2002, pp. 35-40; Ouaknin, 2006, pp. 269-272). Da ciò si può capire come la Cabala, nei secoli successivi, e in particolare nel $\mathrm{XV}$ secolo, assumesse una dimensione di linguaggio magico attraverso cui influire sulla realtà e si sviluppasse poi in svariate applicazioni pratiche, assumendo il carattere di Cabala pratica. Proprio verso la fine del XV secolo, in seguito alla cacciata degli ebrei da Spagna e Portogallo, la cultura cabalistica ebbe modo di propagarsi maggiormente in Europa e in particolare nella penisola italiana. Molti cabalisti di origine ebraica, infatti, si stabilirono in diverse città italiane, tra cui Venezia, Padova, Firenze. Tra questi ve ne furono anche alcuni convertitisi al cristianesimo, come Flavio Mitridate (1445-1489), traduttore dall'ebraico al latino, amico e maestro di Pico della Mirandola (1463-1494), al quale trasmise il suo sapere cabalistico (Secret, 2001, pp. 49-64). Fu proprio Pico della Mirandola a diffondere la Cabala tra gli umanisti cristiani, nell'alveo del neoplatonismo e del cristianesimo. Per Pico, infatti, essa rappresentava uno degli aspetti della prisca theologia, nell'ottica della conciliazione di tutte le filosofie e di tutte le religioni, ed era da lui considerata espressione della rivelazione divina. Pico conosceva gli insegnamenti di Abraham Abulafia e insieme a Marsilio Ficino studiò e diffuse lo Zohar, il più importante testo della tradizione cabalistica. Per l'umanista, che dedicò alla cabala 72 Conclusiones all'interno delle celebri Conclusiones nongentae in omni genere scientiarum (1486), tale dottrina consente di entrare in contatto con il mondo sovraceleste, invocando gli angeli 
e le 10 Sefirot (i 10 nomi o emanazioni di Dio), attraverso il potere della sacra lingua ebraica (Yates, 2019, p. 93). Pico porta la Cabala ebraica nel solco della tradizione cristiana, facendola risalire all'insegnamento di Mosè, considerandola sapienza sacra che conferma la verità del cristianesimo, nonché della divinità di Cristo e della Trinità. Il legame tra Cabala e cristianesimo si esplicita con la raccomandazione di Pico, secondo cui tutte le operazioni intraprese nell'ambito cabalistico devono avere alla base un atteggiamento di pietà e timore verso Dio; la Cabala non è infatti un mero procedimento magico per richiedere l'intervento angelico, ma si riveste di un carattere religioso e mistico, in quanto il momento più importante dell'esperienza cabalistica è l'innalzamento spirituale e la "divinizzazione" del mago, il quale arriva a comprendere in sé le 10 Sefirot, le quali prendono dimora nell'anima, purificandola e rigenerandola (ibid., pp. 109-123).

Se ora guardiamo ad alcuni testi cabalistici di Valetta, possiamo osservare tracce della tradizione cabalistica rinascimentale e al contempo notare una certa semplificazione rispetto alla lezione mistico-magica pichiana, nel cui solco comunque l'autore si pone.

I vari scritti di Valetta sulla cabala hanno un impianto più pratico che mistico, lo spazio dedicato alla disquisizione teorica è alquanto ridotto e si trova in apertura del testo, a guisa di incipit. Possiamo qui soffermarci su uno di questi testi:

Questa Scienza viene chiamata Cabala da queste due decisioni cioè Lab che vuol dire secreto et Ala che vuol dire Dio che viene a dire Secreto di Dio Questa è stata trasportata dall'ebraico in latino, et è quella, con la quale s'interpreta la sua scrittura è fù rivelata da Dio agli Huomini Santissimi et è un modo di rispondere per numeri a qual si sia quesito fatto su cosa naturale presente, passato, e futuro; Mà contingente non introducendo la necessità, essendo sempre conforme la volontà di Dio, et essendo sempre à noi impossibile di saperla, quando vuole, che non siamo degni d'intenderla. (171r)

L'autore, secondo la lezione di Pico della Mirandola, pone la Cabala in seno alla tradizione cristiana e la riconduce direttamente a Dio, dal quale sarebbe stata rivelata agli "Huomini Santissimi", sottolineando 
con ciò allo stesso tempo il suo carattere di dottrina occulta, accessibile soltanto a pochi eletti, che devono custodirla ed esercitarla sempre rispettando la volontà di Dio.

La Cabala ha inoltre uno scopo pratico, ovvero quello di trovare risposte a quesiti riguardanti non solo presente e passato, ma anche il futuro, in ciò allontanandosi dalla lezione pichiana, secondo cui essa era un mezzo per entrare in contatto con gli spiriti angelici e richiedere il loro intervento, nonché per elevare la propria anima; non aveva cioè uno scopo meramente divinatorio (Yates, 2019, pp. 127-128), come pare invece in Valetta. Quest'ultimo si avvale del metodo della gematria, affermando che la Cabala è un "modo di rispondere per numeri a qual si sia quesito", lasciando qui da parte l'arte della permutazione, temura, e dell'anagramma, notarikon, che occupavano invece in Pico un posto rilevante, anche da un punto di vista mistico, inserendosi questi nel solco tracciato da Abraham Abulafia e riprendendo la lezione ermetica della divinizzazione dell'uomo attraverso il congiungimento con Dio (ibid., pp. 106-107).

Sempre considerando l'incipit del testo di Valetta, possiamo notare una corrispondenza con un'altra opera, Opusculum raimundinum de auditu kabbalistico, attribuita a Raimondo Lullo (1232-1316), ma in realtà costituente una trascrizione tarda di un altro testo lulliano, Ars brevis, diffuso a partire dalla fine del XV secolo, in cui erano stati inseriti riferimenti alla Cabala, per rileggere l'opera e il pensiero di Lullo nelle categorie cabalistiche, a partire dall'analogia tra temura e arte combinatoria lulliana (Eco, 2002, p. 141). In apertura dell'opuscolo troviamo questa definizione della dottrina cabalistica: "Secundum modernos Kabbalistas Kabbala cum sit nomen compositum ex duabus dictionibus, videlicet abba et ala. Abba enim arabice idem est quod pater latine, et ala arabice idem est quod Deus meus." (Anonymous, 1578, pp. 3-4), da cui non si discosta molto l'incipit di Valetta. Anch'egli sembrerebbe condividere la rilettura dell'opera lulliana in chiave cabalistica, richiamandosi nel suo incipit alla definizione di Cabala presente nel De audito, con soltanto una lieve modifica nell'interpretazione dell'etimologia della parola, ma conservando il fondamentale riferimento all'origine divina della Cabala, già presente nella sua radice (“Ala”). 
Nel testo di Valetta è inoltre interessante richiamare l'attenzione su di un particolare, ovvero un segno grafico che compare due volte (alle carte $186 \mathrm{v}$ e $187 \mathrm{r}$ ), la cui forma richiama una croce greca con quattro punti, ciascuno dei quali inserito negli spazi delle intersezioni dei bracci della croce stessa (cf. Śnieżyńska-Stolot \& Boni). Parrebbe di poter interpretare tale segno come un simbolo magico che riproduce un amuleto protettivo o un sigillo da mettere in relazione con quelli riprodotti nel Grimorio di Armadel, in particolare con il sigillo per invocare la protezione dell'angelo Thavael ${ }^{9}$. Tale richiamo ci porta a confermare la concezione cabalistica di Valetta sul versante della Cabala pratica, in cui però il riferimento agli spiriti celesti non ha una valenza mistica o di innalzamento dell'anima (come in Pico), ma è da intendere come invocazione diretta per ottenere protezione o l'esaudimento di qualche richiesta, nel caso in questione la risposta a "qual si sia quesito fatto su cosa naturale presente, passato, e futuro". Dalla presenza di tale simbolo possiamo supporre che nel bagaglio di conoscenze del veneziano vi fossero quindi anche quei libri di magia che promettevano, attraverso l'uso dei talismani, di entrare in contatto con potenze spirituali superiori. Oltre al citato Grimorio di Armadel, infatti, anche il Picatrix latino (circolante a partire dal XII secolo, tradotto dall'arabo) basava le operazioni magiche sull'utilizzo di amuleti e talismani, così come la Clavicula Salomonis, altro manuale magico in circolazione dalla metà del XVII secolo, dedicato all'evocazione degli spiriti ultraterreni. Ca-

9 Con grimorio si indica in generale un libro di magia che fornisce istruzioni per invocare spiriti attraverso rituali e formule fisse che mescolano elementi della religione cristiana e della tradizione ebraica, ma anche greca ed egizia. Tra i metodi per ottenere la protezione o l'intervento di determinati spiriti celesti vi è proprio quello della costruzione di amuleti o sigilli con simboli e caratteri legati, ad esempio, all'angelo che si vuole evocare. I grimori tramanderebbero, secondo la tradizione, la sapienza magica del Re Salomone (Guiley, 2006, pp. 121-127). Il Grimorio di Armadel fu pubblicato nel 1625 in latino e forniva un elenco di istruzioni per mettere in atto rituali al fine di evocare determinati spiriti, con l'aiuto di talismani, sigilli, nonché circoli magici. Il sigillo riprodotto da Valetta si trova a p. 14 dell'edizione dell'opera, reperibile in formato elettronico: https:/www.yumpu.com/es/document/read/35378052/macgregormathers-grimorio-de-armadelpdf. Thavael vi è descritto come l'angelo che si prese cura della Sacra Famiglia durante la fuga dalla persecuzione di Erode. 
ratteristica comune di questi testi era lo scopo meramente pratico e immediato da raggiungere, come guarire da una malattia, allungare la vita, trovare tesori, vincere i nemici (Yates, 2019, p. 60). Un'altra prova della conoscenza da parte di Valetta di tale genere di manuali e del suo interesse per tale tipo di pratiche, proviene da una preghiera-invocazione posta a conclusione di un altro testo cabalistico, quello in cui si spiega il modo, sempre attraverso la tecnica della gematria combinata a calcoli astrologici, per conoscere il nome del proprio genio tutelare, Trattato Per estrarre il nome del Genio (106r-113r):

Omnipotens Eterne Deus, qui totam Creaturam condidisti in laudem tuam, et honorem tuum ac ministerium hominis: Oro ut Spiritum et Genium meum bonum N. emittere digneris, ut mè doceat que Illum cum Iustitia et Pietate interrogavero, Verum non mea voluntas, sed tua fiat per Jesum Christum felicissimum Dominum nostrum Amen. (113r)

Si tratta infatti della trascrizione dell'orazione posta nelle prime pagine del Grimorio di Armadel, da recitare per evocare lo spirito, pronunciando il suo nome e tracciando il sigillo che lo riguarda (Anonymous, 1625, p. 10).

Valetta pare condividere la concezione strettamente pragmatica che delle operazioni magiche proponevano questi testi. Si è lontani dalla raffinata magia religioso-filosofica di Pico della Mirandola in cui attraverso la sacra lingua ebraica si invocavano i nomi di Dio (le 10 Sefirot) e Dio stesso. Pico, inoltre, aveva marcato la distanza della sua cabala dalla magia degradata, che storpiava la lingua ebraica e i nomi divini, diffusa sotto il nome di Salomone e a cui appartenevano i testi sopra menzionati (Yates, 2019, p. 119). La lontananza dalla concezione pichiana della cabala si può notare inoltre leggendo uno dei quesiti, invero alquanto prosaico, che Valetta propone come esempio, specificando al contempo che esso deve sempre essere "breve, chiaro, non equivoco, né ambiguo, uno solo", a cui trovare la risposta: "Voglio sapere il nome di quello rubbò li pendenti lenzuoli, et altre robbe tre Anni sono in circa della signora Doralice Fontana" (216v).

Avviandoci alla conclusione, possiamo affermare che gli interessi di Valetta si orientano su due versanti delle scienze occulte (nel sen- 
so di nascoste e sconosciute ai non iniziati): magia naturale e cabala. Le due autorità in tali dottrine sono rispettivamente Giovanni Battista Della Porta e Pico della Mirandola. Per quanto riguarda il primo ambito, quello della magia naturale, Valetta segue da vicino l'insegnamento dellaportiano, dimostrando di condividerne non solo gli aspetti tecnicopratici (importanza della distillazione e della pesatura per giungere alla "quintessenza"), ma anche quelli teorici (principi della concatenazione, dell'accordo e disaccordo tra le cose).

Nel secondo ambito, invece, notiamo un certo discostamento dalla raffinata cabala pichiana, della quale vengono ignorati gli aspetti filosofici e religiosi, che per il pensatore rinascimentale rivestivano una primaria importanza e costituivano i fondamenti del suo pensiero. La Cabala "alta" di Pico della Mirandola subisce in Valetta una "degradazione" a dottrina di uso pratico, potremmo dire di utilizzo quotidiano, per risolvere questioni estremamente concrete, assai poco interessata all'innalzamento dell'adepto ${ }^{10}$. Valetta condivide con Pico l'atteggiamento di timore e pietà verso Dio, solo col Suo volere possono essere intraprese le operazioni cabalistiche, e mette in relazione la Cabala con la tradizione cristiana; tuttavia è assente qualsiasi disegno di dignificazione dell'uomo mago-cabalista, che attraverso la Cabala giunge alla purificazione spirituale e alla contemplazione di Dio. Valetta rimane piuttosto

10 Tale approccio pratico e "prosaico" alla dottrina cabalistica è del resto condiviso dalla stessa Maria Casimira, proprietaria di quelle carte. Ella si serve dei calcoli cabalistici per ottenere risposte a questioni legate alla sua vita o a quella dei suoi familiari (in particolare del figlio Jakub), per conoscere la causa di qualche avvenimento passato o il futuro. L'argomento meriterebbe ampio spazio (per cui si rimanda a ŚnieżyńskaStolot \& Boni); in questa sede si può accennare ad alcuni esempi di domande a cui la regina cerca risposta tramite il metodo cabalistico della gematria (lo stesso esposto da Valetta nei testi menzionati), i quali possono fornire un'idea dell'utilizzo che Marysieńka faceva di questi appunti: “Dica quale sia l'indispositione del tumido Ventre di Giacomo Sobieschi ... [risposta] E' perche senza moto il corpo duro divien, e dal butiro ancora ardente." (159v); "Dica che significa il segno nero nell ongia del deto grosso del piede sinistro di Giacomo Sobieschi ... [risposta] Ogni sinistro mal gli astri maligni ne terminaro al pie per dolce aspetto" (168r); "Dica chi partorirà la Principessa Elisabetta Neuburg [nuora di Maria Casimira] ... [risposta] Il Ciel li donera maschile il parto e del [avo] paterno il nome spande" (163v). 
sul versante di quelle pratiche magico-cabalistiche ancora legate a certi tipi di magia pratica, come testimoniato dalla presenza nel suo testo di segni magici, come il sigillo dell'angelo Thavael, e dalla conoscenza del Grimorio di Armadel, appartenente a quella tradizione di testi che Pico considerava esempi di una magia rozza e degradata. Egli sembra quindi ignorare del tutto il messaggio spirituale alla base della Cabala.

Gli scritti del veneziano hanno comunque un doppio valore testimoniale. Da un lato confermano come a Venezia, a fine Seicento e a inizio Settecento, fosse ancora viva la tradizione degli studi e degli interessi magico-cabalistici, già fiorenti del resto nella città lagunare durante il XVI secolo con autori come Francesco Zorzi, Giovan Lorenzo Anania, Guillaume Postel (Secret, 2001, pp. 130-141, 167-178); dall'altro, come questi interessi fossero condivisi dalla stessa regina Maria Casimira, la quale, abbia o meno conosciuto direttamente Andrea Valetta a Venezia, aveva ritenuto i suoi scritti a tal punto utili e interessanti da leggerli e postillarli, facendo in modo di averli e tenendoli con sé nel suo lungo soggiorno romano ${ }^{11}$.

\section{BIBLIOGRAFIA}

Anonymous (1578). Opusculum Raymundinum de auditu kabbalistico sive ad omnes scientia introductorium. Parigi: Aegidium Gorbinum.

Anonymous (1625). Grimorio di Armadel. Retrieved from https://www.yumpu.com/es/document/read/35378052/macgregor-mathers-grimorio-dearmadelpdf

11 Note di mano di Maria Casimira sono infatti negli spazi vuoti dei fogli su cui si trovano gli scritti di Valetta (ad es. alle carte 93v, 121v, 123v i 141r, 224v), cf. Śnieżyńska-Stolot \& Boni. Aperta rimane la questione di come la regina sia entrata concretamente in possesso di queste carte. Si può ipotizzare, vista la provenienza veneziana di Valetta e la vicinanza temporale tra il soggiorno di Maria Casimira a Venezia e gli anni in cui era in vita l'autore dei testi (cf. supra), che ciò sia avvenuto proprio a Venezia, dove la regina potrebbe aver acquistato o commissionato allo stesso gli scritti. La vedova di Jan Sobieski avrebbe tenuto poi queste carte con sé e le avrebbe portate a Roma, ampliandole con altri testi e con appunti di sua mano, sino a formare la raccolta giunta fino a noi. 
Bassani, A. (1700). Viaggio a Roma della Sua Reale Maestà di Maria Casimira, Regina di Polonia. Roma: Stamparia Barberini.

Boy-Żeleński, T. (1937). Marysieńka Sobieska. Lwów-Warszawa: KsiążnicaAtlas.

Camporesi, P. (1997). Il sugo della vita. Simbolismo e magia del sangue. Milano: Garzanti.

Della Porta, G.B. (1563). De furtivis literarum notis vulgo de Ziferis. Napoli: Ioan. Maria Scotus.

Della Porta, G.B. (1584). Magiae naturalis libri XX. Napoli: Horatius Salvianus.

Della Porta, G.B. (1586). De humana physiognomia. Vico Equense: Josephum Cacchium.

Della Porta, G.B. (1609). De distillationibus libri IX. Strasburgo: Lazari Zetzneri.

Della Porta, G.B. (1677). Della magia naturale del Signor Giovanni Battista Della Porta napolitano libri XX. Napoli: Antonio Bulifon.

Dobrowolski, J.A. (1990). Droga przez labirynty magii. Giambattista Della Porta (1535-1615). Warszawa: Państwowe Wydawnictwo Naukowe.

Eco, U. (2002). W poszukiwaniu języka uniwersalnego. Gdańsk-Warszawa: Marabut.

Guiley, E.G. (2006). The Encyclopedia of Magic and Alchemy. New York: Facts On File.

Komaszyński, M. (1995). Piękna królowa Maria Kazimiera d'Arquien-Sobieska. Kraków: Wydawnictwo Literackie.

Ouaknin, M.A (2006). Tajemnice Kabaty. Warszawa: Cyklady.

Pico della Mirandola, G. (1532). Conclusiones nongentae in omni genere scientiarum. Norimberga: Johannes Petreius.

Pietrzak, J. (2016). Księga Kabał Marii Kazimiery Sobieskiej - nieuznany ślad kultury rzymskiego dworu Królowej Wdowy. Studia Wilanowskie, XXIII, 65-82.

Platania, G. (1988). La Polonia nelle carte del cardinale Carlo Barberini protettore del Regno. Accademie e Biblioteche d'Italia, LVI(2), 38-60.

Platania, G. (1995). Maria Casimira Sobieska a Roma. Alcuni episodi del soggiorno romano di una regina di Polonia. In V. De Caprio (Ed.), Effetto Roma. Il viaggio (pp. 8-48). Roma: Istituto Nazionale di Studi Romani.

Platania, G. (2018). Il soggiorno romano di Maria Casimira Sobieska. Eastern European History Review, 1, 9-17.

Secret, F. (2001). I Cabbalisti Cristiani del Rinascimento. Roma: Arkeios. 
Śnieżyńska-Stolot, E., \& Boni, F. (forthcoming). Księga Kabał Królowej Sobieskiej. Teksty wloskie.

Śnieżyńska-Stolot, E. (2017). Wieszczba astrologiczna przy narodzeniu Jakuba Sobieskiego Królewicza. Kraków: Muzeum Narodowe w Krakowie.

Sosnowski, R. (2019). Kultura romańska w Polsce (od średniowiecza do końca XVIII wieku) na podstawie kolekcji rękopisów francuskich $i$ wtoskich $w$ zbiorach Biblioteki Jagiellońskiej. Retrieved from https://rekopisyromanskie.filg.uj.edu.pl

Sholem, G. (2007). Le grandi correnti della mistica ebraica. Torino: Einaudi. Yates, F.A. (2019). Giordano Bruno e la tradizione ermetica. Bari: Laterza.

Wisłocki, W. (1881). Katalog rękopisów Biblioteki Uniwersytetu Jagiellońskiego. Kraków: Biblioteka Jagiellońska.

Riassunto: Il contributo presenta alcuni testi contenuti nel manoscritto 2284 conservato alla Biblioteca Jagellonica di Cracovia, conosciuto come Księga Kabał Królowej Sobieskiej ("Il libro della Cabala della Regina Sobieska"). Si tratta di un insieme di testi inediti a carattere magico, astrologico, cabalistico, raccolti da Maria Casimira Sobieska, durante il suo lungo soggiorno in Italia (16991714). Tra questi ve ne sono alcuni redatti in italiano, molto probabilmente a Venezia, da un abitante della Serenissima che si firma come Andrea Valetta, la cui mano si riconosce in diversi testi. Alcuni di essi riguardano la magia naturale, altri la dottrina cabalistica: Acqua simpatica per scivere [sic] da lontano, Per fare l'acqua ardente, A'far la scrittura simpatica, Altra Acqua simpatica (104r-105v); Modo di scrivere in Zifra senza dar sospetto di Zifra; Tabella della prima scienza numerica (100r); Seconda scienza numerica (100v-101v); Trattato Per estrarre il nome del Genio (106r-113r); Regole di Cabala di Salomone Imparatale divinam[ente] da Dio (216r-222v). L'articolo dimostra come, nel primo caso, l'autore segua gli insegnamenti di G.B. Della Porta e il suo approccio alla magia naturale, mentre nel secondo, pur condividendo con Pico della Mirandola l'interpretazione cristiana della Cabala, ne ignori gli aspetti mistici e spirituali, privilegiando quelli pratici e divinatori.

Parole chiave: Maria Casimira Sobieska, Venezia, Cabala, magia naturale, XVII-XVIII secolo 